\title{
Perché la dialisi peritoneale non cresce in Italia? Le ragioni della stasi e le prospettive di sviluppo
}

\author{
Gianpaolo Amici
}

\author{
Divisione di Nefrologia e Dialisi \\ Ospedale Regionale "Santa Maria dei Battuti", Treviso
}

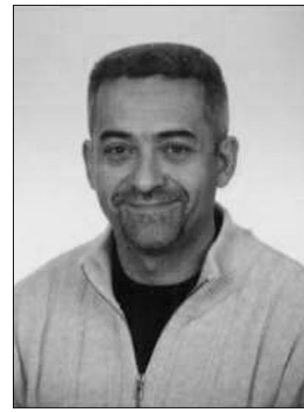

I'

1 Italia è un paese legato alla dialisi peritoneale e ha contribuito in modo rilevante alla sua evoluzione, ma da ormai dieci anni la percentuale di pazienti dializzati che pratica la dialisi peritoneale rimane attorno al $10 \%$. La valutazione delle motivazioni di questa stazionarietà è legata a considerazioni di natura politica, sociale e medica. Le analisi e le motivazioni che seguono hanno un carattere soggettivo per scelta dell'autore e quindi non sono riportati riferimenti bibliografici. Infine le affermazioni seguenti non vogliono assolutamente offendere alcun soggetto sanitario o politico ma vogliono essere solo un momento di riflessione costruttiva.

\section{Considerazioni di natura politica}

La struttura del nostro sistema sanitario è di tipo misto (pubblico e privato convenzionato) con prevalenza di Centri dialisi privati convenzionati al Centro-Sud dove da molti anni le strutture pubbliche non riescono a coprire completamente le esigenze dialitiche della popolazione. Questa organizzazione si fonda sul riconoscimento delle prestazioni con una tariffa. Le tariffe dei vari tipi di dialisi sia extracorporea che peritoneale seguono il principio fondamentale del riconoscimento economico secondo la complessità tecnologica. Questo sistema penalizza la dialisi peritoneale in quanto dialisi autogestita e generalmente ritenuta a bassa tecnologia con tariffe modeste in proporzione ai costi vivi. Anzi, la dialisi peritoneale in quanto trattamento più economico e domiciliare, invece di essere incentivata come scelta più valida in tema di gestione delle risorse dialitiche ed economiche, viene rimborsata a tariffe più basse e quindi di fatto disincentivata. Se a questo fatto si aggiunge che le tariffe possono essere modificate a livello regionale, si può comprendere come la realtà dialitica italiana sia variabile. In Italia vi sono dunque zone a elevata penetrazione della dialisi peritoneale dove il sistema sanitario pubblico è più rappresentato e zone a bassa penetrazione dove il sistema sanitario privato convenzionato prevale e la media nazionale della penetrazione non esprime appieno la realtà. Una statistica migliore sarebbe la determinazione della penetrazione della metodica solo nei Centri che la praticano con il calcolo della potenziale espansione o potenziale risparmio di spesa, applicando il valore di penetrazione così ottenuto ai Centri che non la praticano. In tema di valutazione politica generale della struttura del sistema sanitario nazionale, anche se di fronte alla spesso citata limitazione delle risorse disponibili, è necessario tenere conto che lo Stato, convogliando nella Sanità una parte dell'introito proveniente dalla tassazione, offre opportunità di lavoro e guadagno a moltissimi soggetti con una ricaduta sociale di occupazione e ridistribuzione della ricchezza. In questa prospettiva l'emodialisi ospedaliera o privata che sia, anche seoggettivamente costosa, offre una opportunità di lavoro a moltissimi operatori e a varie ditte. La dialisi peritoneale invece modifica questi equilibri e riducendo i costi tecnici e organizzativi ne modifica anche in parte le ricadute sociali. Forse sarebbe opportuno, anche per lo sviluppo della dialisi peritoneale, fare chiarezza in sede politica se nell' equilibrio dinamico tra allocazione delle risorse e spesa dialitica, con l'annesso indotto sociale di redistribuzione della ricchezza, è possibile perseguire una reale riduzione dei costi, specie considerando la costante espansione della popolazione bisognosa di dialisi. 


\section{Considerazioni di natura sociale}

La dialisi peritoneale è una metodica dialitica domiciliare e di conseguenza si deve integrare socialmente con le esigenze dei pazienti e delle loro famiglie. Le difficoltà di natura sociale che ostacolano lo sviluppo di questa dialisi sono legate all'invecchiamento della popolazione generale con conseguente incremento dell'età di inizio del trattamento. Pazienti uremici più anziani significa pazienti meno autonomi, con una serie di menomazioni fisiche legate all'età come riduzione del visus, tremolio delle mani, riduzione della forza muscolare e della capacità di deambulare e con maggiori probabilità di avere patologie invalidanti concomitanti. Infine la struttura familiare in Italia si è modificata con famiglie meno numerose e più anziani che vivono da soli in case più piccole. Queste considerazioni rendono l'applicazione della dialisi peritoneale problematica per carenza di assistenza e di idonei spazi e di conseguenza con impatto sociale più impegnativo dell' emodialisi che viene eseguita e gestita completamente in ambiente ospedaliero con trasporti organizzati e gratuiti. In questo scenario si comprende come, per incentivare la deospedalizzazione dei trattamenti, deve essere offerto un aiuto sociale organizzato con personale disponibile per l'assistenza domiciliare e spazi medici di distretto o di quartiere per l'esecuzione dei trattamenti. In alternativa può essere offerto un incentivo economico ai pazienti che accettano qualsiasi tipo di cura a domicilio, finalizzato al pagamento di assistenza infermieristica e sociale privata o alla creazione di strutture sanitarie autogestite.

\section{Considerazioni di natura medica}

La dialisi peritoneale ha una fama negativa in ambito nefrologico anche a distanza di molti anni dalla sua introduzione su larga scala e nonostante i suoi miglioramenti tecnologici. Le motivazioni sono molteplici e interconnesse tra loro. In primo luogo la metodica ha una durata modesta se comparata all'emodialisi e la sua scarsa durata è condizionata dalla qualità del follow-up medico specifico. Infatti la dialisi peritoneale utilizza una membrana biologica non sostituibile e, in caso di cattiva gestione, fallisce invariabilmente in breve tempo. Le complicanze possibili sono conosciute: peritonite e infezione dell'exit-site, ernie, sclerosi peritoneale e inadeguatezza della dose dialitica. Queste complicanze richiedono metodologia, terapia ed esperienza specifica che devono garantire la massima sicurezza per il paziente. Si comprende che esperienza e competenza si acquisiscono sul campo e quindi non sono compatibili per esempio con la frammentazione della popolazione dialitica in tante piccole realtà e non possono essere surrogate da corsi di aggiornamento o avviamento. Solo una politica di concentrazione dei pazienti in Centri di riferimento accreditati può garantire gli alti livelli di specializzazione e competenza richiesti. Naturalmente le difficoltà di crescita della metodica sono legate alla reale complessità della stessa e il progresso scientifico nella tecnologia dell'accesso peritoneale, nel contenimento delle infezioni e nella composizione delle soluzioni possono dare nuovo impulso a questa tecnica dialitica. Strettamente connesso all'orientamento del Centro è il metodo utilizzato per la valutazione dell'idoneità del paziente alla dialisi peritoneale e per l'informazione che il paziente riceve. In momenti tanto delicati per la vita del paziente anche lievi sfumature negative o informazioni tardive, frettolose e incomplete possono decidere l'espansione o meno della popolazione dialitica peritoneale nei vari Centri.

\section{Conclusioni}

Chi pratica e studia la dialisi peritoneale sa che una crescita della penetrazione della metodica, ancorché desiderabile e desiderata, non è prevedibile a breve termine senza una radicale modifica politica e organizzativa della Sanità e della Ne- frologia italiana. Volendo restringere all'essenziale direi che dopo avere trovato una chiara convergenza di intenti per lo sviluppo della metodica, una via percorribile potrebbe essere l'incentivazione economica realizzabile con diverse strategie per gli enti, per gli operatori e per i pazienti.

amicig@tin.it 